\title{
Défis et possibilités en matière de santé publique rendus possibles grâce aux progrès du traitement des langues naturelles
}

\author{
Oliver Baclic ${ }^{1 *}$, Matthew Tunis ${ }^{1}$, Kelsey Young ${ }^{1}$, Coraline Doan², Howard Swerdfeger², \\ Justin Schonfeld ${ }^{3 *}$
}

\section{Résumé}

Le traitement des langues naturelles (TLN) est un sous-domaine de l'intelligence artificielle consacré à la compréhension et à la création du langage. Les progrès récents des technologies du TLN permettent d'analyser rapidement des textes en grande quantité, créant ainsi des possibilités de recherche en santé et de prise de décisions éclairées par des données probantes. L'analyse et l'extraction de données à partir de la documentation scientifique, des rapports techniques, des dossiers de santé, des médias sociaux, des enquêtes, des registres et d'autres documents peuvent appuyer les fonctions essentielles de la santé publique, notamment l'amélioration des systèmes de surveillance existants (e.g. en identifiant plus rapidement les maladies et les facteurs de risque ou les populations à risque), les stratégies de prévention des maladies (e.g. en évaluant plus efficacement la sécurité et l'efficacité des interventions) et les efforts de promotion de la santé (e.g. en fournissant la capacité d'obtenir des réponses d'expert à toute question liée à la santé). Le TLN est en train de devenir un outil important qui peut aider les autorités de la santé publique à réduire le fardeau de l'inégalité et de l'iniquité en matière de santé parmi la population. Le présent document a pour but de fournir des exemples notables des applications et des défis possibles découlant de l'utilisation du TLN en santé publique.

Citation proposée : Baclic O, Tunis M, Young K, Doan C, Swerdfeger H, Schonfeld J. Défis et possibilités de en matière de santé publique rendus possibles grâce aux progrès du traitement des langues naturelles. Relevé des maladies transmissibles au Canada 2020;46(6):182-90. https://doi.org/10.14745/ccdr.v46i06a02f

Mots-clés : traitement des langues naturelles, TLN, intelligence artificielle, apprentissage automatique, santé publique

\section{Introduction}

On s'intéresse de plus en plus au déploiement de stratégies de l'intelligence artificielle (IA) pour atteindre les résultats en matière de santé publique, en particulier en réponse à la pandémie mondiale de la maladie à coronavirus de 2019 (COVID-19), où de nouveaux ensembles de données, des outils de surveillance et des modèles émergent très rapidement.

L'objectif de ce manuscrit est de fournir un cadre pour l'examen des approches du traitement des langues naturelles (TLN) en matière de santé publique fondées sur des applications historiques. Cet aperçu comprend une brève introduction à I'IA et au TLN, suggère des possibilités où le TLN peut être appliqué pour résoudre les problèmes de santé publique et décrit les défis liés à l'application du TLN dans un contexte de santé publique. Des articles particuliers ont été choisis pour souligner l'ampleur des applications possibles du TLN en santé publique ainsi que les défis et les risques non négligeables inhérents à l'intégration de I'IA et du TLN dans l'analyse de la santé publique et l'appui à la décision.

\section{Intelligence artificielle et traitement des langues naturelles}

La recherche en IA a produit des modèles qui peuvent interpréter une radiographie $(1,2)$, détecter des battements de cœur irréguliers à l'aide d'une montre intelligente (3), identifier automatiquement les rapports de maladies infectieuses dans les médias (4), déterminer les facteurs de risque cardiovasculaire à partir d'images rétiniennes (5) et trouver de nouvelles cibles pour les médicaments existants $(6,7)$. Le succès de ces modèles s'appuie sur l'entraînement de centaines, de milliers 
et parfois de millions de points de données contrôlés, étiquetés et structurés (8). La capacité de l'IA de fournir des analyses constantes, soutenues et rapides des données offre le potentiel de transformer l'approche de la société en matière de promotion de la santé, de prévention et de gestion des maladies. Les systèmes $\mathrm{d}^{\prime}$ IA peuvent « lire » et trier environ tous les 1,3 million d'articles de recherche indexés par PubMed chaque année (9); " examiner » les commentaires de 1,5 milliard d'utilisateurs de Facebook ou « surveiller » 500 millions de gazouillis de personnes souffrant de maladies mentales tous les jours, de maladies d'origine alimentaire ou de grippe $(10,11)$; et interagir en même temps avec chaque personne qui cherche des réponses à ses questions, préoccupations, problèmes et défis en matière de santé (12).

Le TLN est un sous-domaine de l'IA qui se consacre au développement d'algorithmes et à la construction de modèles capables d'utiliser le langage de la même manière que les humains (13). Il est couramment utilisé dans les assistants virtuels comme « Siri » et «Alexa » ou dans les recherches et traductions Google. Le TLN permet d'analyser et d'extraire des renseignements des sources non structurées, d'automatiser la réponse aux questions et de mener une analyse des sentiments et à la synthèse du texte (8). Les langues naturelles (communication) étant le principal moyen de collecte et d'échange de connaissances en santé publique et en médecine, le TLN est la clé pour libérer le potentiel de l'IA dans les sciences biomédicales.

La plupart des plateformes modernes du TLN sont construites sur des modèles affinés au moyen des techniques d'apprentissage automatique $(14,15)$. Les techniques d'apprentissage automatique sont fondées sur quatre composantes : un modèle, des données, une fonction de perte, qui est une mesure qui montre à quel point le modèle convient aux données; et un algorithme pour former (améliorer) le modèle (16). Les récentes percées dans ces domaines ont permis d'améliorer considérablement les modèles TLN qui sont alimentés par un apprentissage profond, un sous-domaine de l'apprentissage automatique (17).

L'innovation dans les différents types de modèles, tels que les modèles basés sur un réseau de neurones récurrents (RNR), les modèles basés sur un réseau de neurones convolutif (RNC) et les modèles axés sur l'attention, a permis aux systèmes du TLN modernes de saisir et de modéliser des relations et des concepts linguistiques plus complexes qu'une simple présence de mots (c'est-à-dire la recherche de mots clés) (18). Cet effort a été soutenu par des approches d'intégration des vecteurs pour traiter à l'avance les données qui encodent les mots avant qu'ils n'alimentent un modèle. Ces approches reconnaissent que les mots existent dans un contexte (e.g. la signification des mots " patient », " tir » et " virus » varient selon le contexte) et les traitent comme des points dans un espace conceptuel plutôt que comme des entités isolées. Le rendement des modèles a également été amélioré par l'avènement du transfert d'apprentissage, c'est-à-dire en prenant un modèle formé pour exécuter une tâche et en l'utilisant comme modèle de départ pour la formation sur une tâche connexe. Les progrès réalisés en matière de matériels et l'augmentation des ensembles de données gratuites et accessibles annotées ont également amélioré les rendements des modèles de TLN. De nouveaux outils d'évaluation et de mises au banc d'essai, tels que GLUE, superglue et $\mathrm{BioASQ}$, nous aident à mieux comprendre le type et la portée de l'information que ces nouveaux modèles peuvent saisir (19-21).

\section{Possibilités}

La santé publique vise à atteindre des résultats optimaux en matière de santé au sein des différentes populations et entre elles, principalement en élaborant et en mettant en œuvre des interventions qui ciblent les causes modifiables de la mauvaise santé (22-26). Le succès dépend de la capacité de quantifier efficacement le fardeau des maladies ou des facteurs de risque de maladies au sein de la population et d'identifier ensuite les groupes qui sont touchés de manière disproportionnée ou à risque, d'identifier des pratiques exemplaires (c.-à-d. des stratégies de prévention optimale ou thérapeutiques) et évaluer les résultats (27). Ce modèle de prise de décision fondé sur des données probantes est mieux représenté par le concept de $\mathrm{PICO}$ (patient/problème, intervention/exposition, comparaison, résultat). PICO fournit une stratégie optimale d'identification des connaissances pour encadrer des questions précises cliniques ou relatives à la santé publique et y répondre (28). La prise de décisions fondées sur des données probantes est généralement fondée sur un examen et une synthèse exhaustifs et systématiques des données conformément aux éléments du cadre de PICO.

Aujourd'hui, l'information est produite et publiée (e.g. la documentation scientifique, les rapports techniques, les dossiers de santé, les médias sociaux, les enquêtes, les registres et d'autres documents) à un rythme sans précédent. En fournissant la capacité d'analyser rapidement des textes non structurés ou semi-structurés en grande quantité, le TLN ouvre d'immenses possibilités de recherches textuelles et de prise de décisions fondées sur des données probantes (29-34). Le TLN est en train de devenir un outil potentiellement puissant pour appuyer I'identification rapide des populations, des interventions et des résultats d'intérêt qui sont nécessaires à la surveillance des maladies, à la prévention des maladies et à la promotion de la santé. Par exemple, l'utilisation de plateformes de TLN capables de détecter des caractéristiques particulières d'individus (population/problème, par exemple, un état de santé ou un facteur prédisposant de risque biologique, comportemental, environnemental ou socio-économique) dans des dossiers médicaux non structurés ou des textes sur les médias sociaux peut être utilisée pour améliorer les systèmes de surveillance existants avec des données probantes réelles. Une étude récente a démontré la capacité des méthodes de TLN à prédire 
la présence de dépression avant son apparition dans le dossier médical (35). La capacité de procéder à l'extraction en temps réel de textes de publications scientifiques à la recherche d'un concept particulier de PICO donne aux décideurs l'occasion de formuler rapidement des recommandations sur la prévention ou la gestion des maladies qui sont fondées sur les données probantes les plus récentes lorsqu'il est essentiel de fournir des conseils en temps opportun, par exemple pendant une éclosion. Les plateformes de question-réponse et les robots conversationnels de TLN sont également susceptibles d'améliorer les activités de promotion de la santé en faisant participer des personnes et en fournissant un soutien ou des conseils personnalisés. Le tableau 1 donne des exemples d'applications potentielles du TLN en santé publique qui ont démontré au moins un certain succès.

\section{Tableau 1 : Exemples d'applications existantes et potentielles du traitement des langues naturelles en santé publique}

\begin{tabular}{|c|c|c|}
\hline Type d'activité & $\begin{array}{l}\text { Objectif en matière } \\
\text { de santé publique }\end{array}$ & $\begin{array}{l}\text { Exemple d'utilisation du } \\
\text { TLN }\end{array}$ \\
\hline \multirow[t]{2}{*}{$\begin{array}{l}\text { Identification } \\
\text { des populations } \\
\text { à risque ou } \\
\text { des conditions } \\
\text { d'intérêt }\end{array}$} & $\begin{array}{l}\text { Mesurer en } \\
\text { permanence l'incidence } \\
\text { et la prévalence } \\
\text { des maladies et des } \\
\text { facteurs de risque } \\
\text { des maladies (e.g. la } \\
\text { surveillance) }\end{array}$ & $\begin{array}{l}\text { Analyse de textes non } \\
\text { structurés ou semi-structurés } \\
\text { tirés de dossiers de santé } \\
\text { électroniques ou de médias } \\
\text { sociaux (36-42) }\end{array}$ \\
\hline & $\begin{array}{l}\text { Identifier les } \\
\text { populations vulnérables } \\
\text { et à risque }\end{array}$ & $\begin{array}{l}\text { Analyse des comportements } \\
\text { à risque à l'aide des médias } \\
\text { sociaux (43-45) }\end{array}$ \\
\hline \multirow[t]{2}{*}{$\begin{array}{l}\text { Détermination } \\
\text { des interventions } \\
\text { médicales }\end{array}$} & $\begin{array}{l}\text { Élaborer des } \\
\text { recommandations } \\
\text { ou des interventions } \\
\text { optimales }\end{array}$ & $\begin{array}{l}\text { Examen et analyse } \\
\text { systématiques automatisés } \\
\text { des renseignements } \\
\text { contenus dans les } \\
\text { publications scientifiques et } \\
\text { les données non publiées } \\
\text { (46-50) }\end{array}$ \\
\hline & $\begin{array}{l}\text { Déterminer les } \\
\text { pratiques exemplaires }\end{array}$ & $\begin{array}{l}\text { Identification des } \\
\text { interventions prometteuses } \\
\text { en matière de santé } \\
\text { publique au moyen de } \\
\text { l'analyse de la littérature } \\
\text { grise ou celle revue par les } \\
\text { pairs disponible en ligne (51) }\end{array}$ \\
\hline \multirow[t]{2}{*}{$\begin{array}{l}\text { Identification } \\
\text { des résultats } \\
\text { en matière de } \\
\text { santé à l'aide } \\
\text { de données } \\
\text { probantes réelles }\end{array}$} & $\begin{array}{l}\text { Évaluer les avantages } \\
\text { des interventions } \\
\text { médicales }\end{array}$ & $\begin{array}{l}\text { Analyse de textes non } \\
\text { structurés ou semi-structurés } \\
\text { tirés de dossiers de santé } \\
\text { électroniques, de médias } \\
\text { en ligne et de publications } \\
\text { pour déterminer l'incidence } \\
\text { des recommandations et des } \\
\text { interventions en matière de } \\
\text { santé publique }(52,53)\end{array}$ \\
\hline & $\begin{array}{l}\text { Déterminer les résultats } \\
\text { négatifs imprévus liés } \\
\text { aux interventions }\end{array}$ & $\begin{array}{l}\text { Analyse de textes non } \\
\text { structurés ou semi- } \\
\text { structurés tirés de dossiers } \\
\text { de santé électroniques, } \\
\text { de médias sociaux et de } \\
\text { publications pour identifier } \\
\text { les événements indésirables } \\
\text { potentiels découlant des } \\
\text { interventions (54-58) }\end{array}$ \\
\hline
\end{tabular}

Tableau 1 : Exemples d'applications existantes et potentielles du traitement des langues naturelles en santé publique (suite)

\begin{tabular}{|c|c|c|}
\hline $\begin{array}{c}\text { Type } \\
\text { d'activité }\end{array}$ & $\begin{array}{c}\text { Objectif en } \\
\text { matière de santé } \\
\text { publique }\end{array}$ & $\begin{array}{c}\text { Exemple d'utilisation } \\
\text { du TLN }\end{array}$ \\
\hline \multirow[t]{2}{*}{$\begin{array}{l}\text { Production et } \\
\text { transfert des } \\
\text { connaissances }\end{array}$} & $\begin{array}{l}\text { Appuyer la recherche } \\
\text { en santé publique }\end{array}$ & $\begin{array}{l}\text { Analyse et extraction } \\
\text { des renseignements tirés } \\
\text { des dossiers de santé } \\
\text { électroniques et des } \\
\text { publications scientifiques } \\
\text { aux fins de la production de } \\
\text { connaissances (59-62) }\end{array}$ \\
\hline & $\begin{array}{l}\text { Appuyer le processus } \\
\text { décisionnel fondé } \\
\text { sur des données } \\
\text { probantes }\end{array}$ & $\begin{array}{l}\text { Utilisation de robots } \\
\text { conversationnels, de } \\
\text { systèmes de questions- } \\
\text { réponses et de résumés } \\
\text { de textes pour fournir } \\
\text { des renseignements } \\
\text { personnalisés aux } \\
\text { personnes qui demandent } \\
\text { des conseils pour améliorer } \\
\text { leur santé et prévenir des } \\
\text { maladies (63-65) }\end{array}$ \\
\hline \multirow[t]{2}{*}{$\begin{array}{l}\text { Analyse de } \\
\text { l'environnement } \\
\text { et connaissance } \\
\text { de la situation }\end{array}$} & $\begin{array}{l}\text { Procéder à des } \\
\text { évaluations des } \\
\text { risques pour la santé } \\
\text { publique et fournir } \\
\text { une connaissance de la } \\
\text { situation }\end{array}$ & $\begin{array}{l}\text { Analyse du contenu en } \\
\text { ligne pour la détection } \\
\text { et l'atténuation des } \\
\text { événements critiques en } \\
\text { temps réel (66-70) }\end{array}$ \\
\hline & $\begin{array}{l}\text { Surveiller les activités } \\
\text { qui peuvent avoir } \\
\text { une incidence sur la } \\
\text { prise de décisions } \\
\text { en matière de santé } \\
\text { publique }\end{array}$ & $\begin{array}{l}\text { Analyse des décisions des } \\
\text { intervenants nationaux et } \\
\text { internationaux (71) }\end{array}$ \\
\hline
\end{tabular}

Abréviation : TLN, traitement des langues naturelles

\section{Défis}

Malgré les progrès récents, il reste encore des obstacles qui empêchent l'utilisation généralisée des technologies de TLN.

À l'instar d'autres techniques d'intelligence artificielle, le TLN dépend fortement de la disponibilité, de la qualité et de la nature des données d'entraînement (72). L'accès et la disponibilité des ensembles de données annotées de façon appropriée (pour utiliser efficacement l'apprentissage supervisé ou semi-surveillé) sont essentiels à la formation et à la mise en œuvre de modèles de TLN robustes. Par exemple, l'élaboration et l'utilisation d'algorithmes capables de procéder à une synthèse systématique de la recherche publiée sur un sujet particulier ou à une analyse et une extraction de données des dossiers de santé électroniques nécessitent un accès illimité aux bases de données des éditeurs ou des soins primaires et des hôpitaux. Bien que le nombre d'ensembles de données biomédicales gratuits et accessibles et de modèles préalablement entraînés ait augmenté ces dernières années, la disponibilité de ceux qui traitent des concepts de santé publique demeure limitée (73).

La capacité d'éviter une distorsion à l'égard des données (c.-à-d. en fournissant la capacité d'inspecter, d'expliquer et d'ajuster 
les données d'un point de vue éthique) représente un autre point important de la formation et de l'utilisation des modèles du TLN dans le secteur de la santé publique à prendre en considération. Le fait de ne pas tenir compte des distorsions lors de l'élaboration (e.g. annotation des données), du déploiement (e.g. utilisation de plateformes préalablement formées) et de l'évaluation des modèles de TLN pourrait compromettre les extrants du modèle et renforcer l'iniquité existante en matière de santé (74). Toutefois, il est important de noter que même lorsque les ensembles de données et les évaluations sont ajustés en fonction des distorsions, cela ne garantit pas les mêmes incidences dans l'ensemble des strates moralement pertinentes. Par exemple, I'utilisation des données sur la santé disponibles sur les plateformes de médias sociaux doit prendre en compte des groupes d'âge et des groupes socio-économiques précis qui les utilisent. Un système de surveillance entraîné sur les données de Facebook est susceptible d'être biaisé au profit des données de santé et des bizarreries linguistiques spécifiques à une population plus âgée comparé à un système entraîné sur les données de Snapchat (75). Récemment, de nombreux outils élaborés suivant un modèle agnostique sont conçus pour évaluer et corriger l'inégalité dans les modèles d'apprentissage automatique, conformément aux efforts déployés par le gouvernement et les milieux universitaires afin de définir le développement inacceptable de l'intelligence artificielle (76-81).

À l'heure actuelle, l'un des principaux obstacles au développement des systèmes de TLN en santé publique est I'accès limité aux données $(82,83)$. Au Canada, les données sur la santé sont généralement contrôlées à l'échelle régionale et, en raison de problèmes de sécurité et de confidentialité, on hésite à fournir un accès illimité à ces systèmes et à les intégrer à d'autres ensembles de données (e.g. couplage de données). Il y a aussi des défis à relever en ce qui concerne la perception du public de la protection de la vie privée et de l'accès aux données. Une récente enquête auprès des utilisateurs des médias sociaux a révélé que la majorité d'entre eux considérait que l'analyse de leurs données sur les médias sociaux pour identifier des problèmes de santé mentale était " gênante et exposante » et qu'ils n'approuveraient pas cela (84).

Avant que des activités de santé publique clés relatives au TLN ne puissent être réalisées à grande échelle, comme l'analyse en temps réel des tendances nationales des maladies, les administrations devront déterminer conjointement une portée raisonnable et l'accès aux sources de données pertinentes à la santé publique (e.g. les dossiers de santé et les données administratives). Afin de prévenir les violations de la vie privée et I'utilisation abusive des données, les applications futures du TLN dans l'analyse des données personnelles sur la santé dépendent de la capacité d'intégrer la confidentialité différentielle dans les modèles (85), pendant la période de formation et l'aprèsdéploiement. L'accès aux données importantes est également limité par les méthodes actuelles, qui permettent l'accès aux publications des textes intégraux. La réalisation de l'extraction et de la synthèse des connaissances entièrement automatisées propres à $\mathrm{PICO}$ exigera un accès illimité aux bases de données des journaux ou à de nouveaux modèles de stockage des données (86).

Enfin, comme pour toute nouvelle technologie, il faut tenir compte de l'évaluation et de l'évaluation des modèles de TLN pour s'assurer qu'ils fonctionnent comme prévu et qu'ils suivent le rythme auquel les opinions éthiques de la société évoluent. Ces technologies de TLN doivent être évaluées pour s'assurer qu'elles fonctionnent comme prévu et qu'elles tiennent compte des distorsions (87). Bien qu'aujourd'hui de nombreuses approches affichent des scores équivalents ou supérieurs à ceux de l'homme sur les tâches d'analyse textuelle, il est important de ne pas assimiler les scores élevés à la compréhension réelle de la langue. Il est toutefois tout aussi important de ne pas considérer le manque de compréhension réelle d'une langue comme un manque d'utilité. Les modèles ayant une compréhension " relativement faible " peuvent encore être très efficaces pour les tâches d'extraction, de classification et de prédiction des renseignements, en particulier avec la disponibilité croissante des données étiquetées.

\section{Traitement des langues naturelles et la maladie à coronavirus de 2019 (COVID-19)}

Depuis l'apparition de la COVID-19, le TLN a joué un rôle primordial dans les efforts fournis lors de l'intervention pour lutter contre les éclosions $(88,89)$. Le TLN a été rapidement utilisé pour analyser la grande quantité de renseignements textuels qui ont été consultés au moyen de l'accès illimité aux journaux, aux prépublications et aux médias numériques revus par les pairs (90). Le TLN a été largement utilisé pour aider les communautés médicale et scientifique à trouver des réponses aux questions de recherche clés, à résumer les données probantes, à répondre aux questions, à suivre la désinformation et à surveiller le sentiment de la population (91-97).

\section{Conclusion}

Le TLN crée des occasions extraordinaires d'améliorer la prise de décisions éclairées par des données probantes en santé publique. Nous prévoyons que des applications plus larges du TLN mèneront à la création de systèmes de surveillance plus efficaces, qui seront en mesure d'identifier les maladies et les conditions à risque en temps réel. De même, grâce à la capacité d'analyser et de synthétiser de grands volumes de renseignements presque instantanément, le TLN doit faciliter des activités ciblées de promotion de la santé et de prévention des maladies, ce qui entraînera possiblement une réduction des maladies à l'échelle de la population et une plus grande équité en matière de santé. Cependant, ces possibilités ne sont pas sans risques : les modèles biaisés, les données biaisées, la perte de la confidentialité de données et la nécessité de maintenir et de mettre à jour les modèles pour refléter l'évolution de la 
langue et du contexte de la communication publique présentent tous des défis qui existent déjà et qui devront être relevés. Nous encourageons les communautés de la santé publique et de la science informatique à collaborer afin d'atténuer ces risques, de veiller à ce que la pratique de la santé publique ne prenne pas de retard par rapport à ces technologies ou rate les opportunités de promotion de la santé, ainsi que de surveillance et de prévention des maladies dans ce paysage en évolution rapide.

\section{Déclaration des auteurs}

O. B. - Rédaction - ébauche initiale, révision et modification, et conceptualisation

M. T. - Rédaction - ébauche initiale, révision et modification, et conceptualisation

K. Y. - Rédaction - révision et modification, et

conceptualisation

C. D. - Rédaction - révision et modification

H. S. - Rédaction - révision et modification

J. S. - Rédaction - ébauche initiale, révision et modification, et conceptualisation

\section{Conflits d'intérêts}

Aucun.

\section{Remerciements}

Nous remercions J. Nash et J. Robertson qui ont eu la gentillesse $d^{\prime}$ 'offrir leurs commentaires et leurs suggestions.

\section{Financement}

Ce travail est appuyé par l'Agence de la santé publique du Canada. La recherche entreprise par J. S. a été financée par I'Initiative de recherche et de développement en génomique du gouvernement fédéral canadien.

\section{Références}

1. Majkowska A, Mittal S, Steiner DF, Reicher JJ, McKinney SM, Duggan GE, Eswaran K, Cameron Chen PH, Liu Y, Kalidindi SR, Ding A, Corrado GS, Tse D, Shetty S. Chest radiograph interpretation with deep learning models: assessment with radiologist-adjudicated reference standards and population-adjusted evaluation. Radiology 2020;294(2):421-31. DOI PubMed

2. Liu X, Faes L, Kale A, Wagner SK, Fu DJ, Bruynseels A, Mahendiran T, Moraes G, Shamdas M, Kern C, Ledsam JR, Schmid MK, Balaskas K, Topol EJ, Bachmann LM, Keane PA Denniston AK. A comparison of deep learning performance against health care professionals in detecting diseases from medical imaging: a systematic review and meta-analysis. Lancet Digital Health 2019. DOI

3. Perez MV, Mahaffey KW, Hedlin H, Rumsfeld JS, Garcia A, Ferris T, Balasubramanian V, Russo AM, Rajmane A, Cheung
L, Hung G, Lee J, Kowey P, Talati N, Nag D, Gummidipundi SE, Beatty A, Hills MT, Desai S, Granger CB, Desai M, Turakhia MP; Apple Heart Study Investigators. Large-scale assessment of a smartwatch to identify atrial fibrillation. $\mathrm{N}$ Engl J Med 2019;381(20):1909-17. DOI PubMed

4. Feldman J, Thomas-Bachli A, Forsyth J, Patel ZH, Khan K. Development of a global infectious disease activity database using natural language processing, machine learning, and human expertise. J Am Med Inform Assoc 2019;26(11):13559. DOI PubMed

5. Poplin R, Varadarajan AV, Blumer K, Liu Y, McConnell MV, Corrado GS, Peng L, Webster DR. Prediction of cardiovascular risk factors from retinal fundus photographs via deep learning. Nat Biomed Eng 2018;2(3):158-64. DOI PubMed

6. Vamathevan J, Clark D, Czodrowski P, Dunham I, Ferran E, Lee G, Li B, Madabhushi A, Shah P, Spitzer M, Zhao S. Applications of machine learning in drug discovery and development. Nat Rev Drug Discov 2019;18(6):463-77. DOI PubMed

7. Corsello SM, Nagari RT, Spangler RD, Rossen J, Kocak M, Bryan JG, Humeidi R, Peck D, Wu X, Tang AA, Wang VM, Bender SA, Lemire E, Narayan R, Montgomery $P$, Ben-David U, Garvie CW, Chen Y, Rees MG, Lyons NJ, McFarland JM, Wong BT, Wang L, Dumont N, O'Hearn PJ, Stefan E, Doench JG, Harrington CN, Greulich $\mathrm{H}$, Meyerson M, Vazquez F, Subramanian A, Roth JA, Bittker JA, Boehm JS, Mader CC, Tsherniak A, Golub TR. Discovering the anticancer potential of non-oncology drugs by systematic viability profiling. Nat Can 2020;1:235-48. DOI

8. Topol EJ. High-performance medicine: the convergence of human and artificial intelligence. Nat Med 2019 Jan;25(1):44-56. DOI PubMed

9. MEDLINE PubMed Production Statistics. Bethesda (MD): U.S. National Library of Medicine (mise à jour 2019-11-19; accédé 2020-01-27). https://www.nlm.nih.gov/bsd/medline_ pubmed_production_stats.html

10. Twitter usage statistics. Internet LiveStats.com (mise à jour 2013-08-16; accédé 2020-01-27). https://www. internetlivestats.com/twitter-statistics/

11. Searching for health. Google News Lab, Schema; 2017 (accédé 2020-01-27). https://googlenewslab.gistapp.com/ searching-for-health

12. Friedman $C$, Elhadad N. Natural language processing in health care and biomedicine. In: Shortliffe E, Cimino J, editors. Biomed Informatics London: Springer; 2014. DOI

13. Ruder S. NLP-progress. London (UK): Sebastian Ruder (accédé 2020-01-18). https://nlpprogress.com/

14. Jurafsky D, Martin JH. Speech and language processing. Stanford (CA): Stanford University; 2019 (mise à jour 2019-11-16; accédé 2020-01-18). https://web.stanford. edu/ jurafsky/slp3/ 
15. Nadkarni PM, Ohno-Machado L, Chapman WW. Natural language processing: an introduction. J Am Med Inform Assoc 2011;18(5):544-51. DOl PubMed

16. Nilsson N. Introduction to machine learning. Stanford (CA): Robotic Library, Department of Computer Science, Stanford University; 1998. http://robotics.stanford.edu/people/ nilsson/MLBOOK.pdf

17. Zhou M, Duan N, Liu S, Shum HY. Progress in neural NLP: modeling, learning, and reasoning. Engineering 2020;6(3):275-90. DOI

18. Tang B, Pan Z, Yin K, Khateeb A. Recent advances of deep learning in bioinformatics and computational biology. Front Genet 2019;10:214. DOI PubMed

19. Hirschberg J, Manning CD. Advances in natural language processing. Science 2015;349(6245):261-6. DOI PubMed

20. Wang A, Singh A, Michael J, Hill F, Levy O, Bowman S. GLUE: a multi-task benchmark and analysis platform for natural language understanding. Proceedings of the 2018 EMNLP Workshop BlackboxNLP: Analyzing and Interpreting Neural Networks for NLP. Brussels (BE): 2018 Nov; p. 353-5. DOI

21. The Big Bad NLP Database. New York (NY): Quantum Stat; 2020 (mise à jour 2020-01-21; accédé 2020-01-27). https://quantumstat.com/dataset/dataset.html

22. Jackson B, Huston P. Promouvoir l'équité en santé en vue d'améliorer la santé : le moment d'agir est venu. Promotion de la santé et prévention des maladies chroniques au Canada 2016;36(2):17-21. DOI

23. Pan American Health Organization. Just societies: health equity and dignified lives. Report of the Commission of the Pan American Health Organization on Equity and Health Inequalities in the Americas. Washington (DC): Pan American Health Organization; 2019-10-01 (mise à jour 2019-11; accédé 2020-01-18). http://search.ebscohost.com/login.aspx ?direct $=$ true \&site $=$ eds-live $\& \mathrm{db}=$ edsebk $\& A N=2329553$

24. Marmot M, Allen J, Goldblatt P, Boyce T, McNeish D, Grady M, Geddes I; The Marmot Review. Fair society, healthy lives: strategic review of health inequalities in England post-2010. UCL Institute of Health Equity. http://www.parliament.uk/documents/fair-society-healthy-live s-full-report.pdf

25. Arcaya MC, Arcaya AL, Subramanian SV. Inequalities in health: definitions, concepts, and theories. Glob Health Action 2015;8:27106. DOI PubMed

26. Agence de la santé publique du Canada. Rapport de L'administrateur en chef de la santé publique sur l'état de la santé publique au Canada 2008 - S'attaquer aux inégalités en santé. Ottawa $(\mathrm{ON})$ : Agence de la santé publique du Canada; 2008. Rapport No.: HP2-10/2008F. https://www. canada.ca/content/dam/phac-aspc/migration/phac-aspc/ cphorsphc-respcacsp/2008/fr-rc/pdf/CPHO-Report-f.pdf
27. Ndumbe-Eyoh S, Dyck L, Clement C. Common agenda for public health action on health equity. Antigonish (NS): National Collaborating Centre for Determinants of Health, St Francis Xavier University; 2016 http://nccdh.ca/images/ uploads/comments/Common_Agenda_EN.pdf

28. Alonso-Coello P, Schünemann HJ, Moberg J, Brignardello-Petersen R, Akl EA, Davoli M, Treweek S, Mustafa RA, Rada G, Rosenbaum S, Morelli A, Guyatt GH, Oxman AD; GRADE Working Group. GRADE Evidence to Decision (EtD) frameworks: a systematic and transparent approach to making well informed healthcare choices. 1: Introduction. BMJ 2016;353:i2016. DOI PubMed

29. Kim ES, James P, Zevon ES, Trudel-Fitzgerald C, Kubzansky LD, Grodstein F. Social media as an emerging data resource for epidemiologic research: characteristics of social media users and non-users in the Nurses' Health Study II. Am J Epidemiol 2020;189(2):156-61. DOI PubMed

30. Koleck TA, Dreisbach C, Bourne PE, Bakken S. Natural language processing of symptoms documented in free-text narratives of electronic health records: a systematic review. J Am Med Inform Assoc 2019;26(4):364-79. DOI PubMed

31. Marshall IJ, Wallace BC. Toward systematic review automation: a practical guide to using machine learning tools in research synthesis. Syst Rev 2019;8(1):163. DOl PubMed

32. Yin Z, Sulieman LM, Malin BA. A systematic literature review of machine learning in online personal health data. J Am Med Inform Assoc 2019;26(6):561-76. DOI PubMed

33. Kreimeyer K, Foster M, Pandey A, Arya N, Halford G, Jones SF, Forshee R, Walderhaug M, Botsis T. Natural language processing systems for capturing and standardizing unstructured clinical information: A systematic review. J Biomed Inform 2017:73:14-29. DOI PubMed

34. The Office of the National Coordinator for Health Information Technology. Health IT dashboard: Quick stats. Washington (DC): U.S. Department of Health and Human Services. https://dashboard.healthit.gov/quickstats/ quickstats.php

35. Harris JK, Mansour R, Choucair B, Olson J, Nissen C, Bhatt J; Centers for Disease Control and Prevention. Health department use of social media to identify foodborne illness - Chicago, Illinois, 2013-2014. MMWR Morb Mortal Wkly Rep 2014;63(32):681-5. PubMed

36. Gesualdo F, Stilo G, Agricola E, Gonfiantini MV, Pandolfi E, Velardi P, Tozzi AE. Influenza-like illness surveillance on Twitter through automated learning of naïve language. PLoS One 2013;8(12):e82489. DOl PubMed

37. Eichstaedt JC, Smith RJ, Merchant RM, Ungar LH, Crutchley P, Preoțiuc-Pietro D, Asch DA, Schwartz HA. Facebook language predicts depression in medical records. Proc Natl Acad Sci USA. 2018;115(44):11203-8. DOI

38. Şerban O, Thapen N, Maginnis B, Hankin C, Foot V. Real-time processing of social media with SENTINEL: a syndromic surveillance system incorporating deep learning for health classification. Inf Process Manage 2019;56(3):1166-84. DOl 
39. Edo-Osagie O, Smith G, Lake I, Edeghere O, De La Iglesia B. Twitter mining using semi-supervised classification for relevance filtering in syndromic surveillance. PLoS One 2019;14(7):e0210689. DOI PubMed

40. Ford E, Carroll JA, Smith HE, Scott D, Cassell JA. Extracting information from the text of electronic medical records to improve case detection: a systematic review. J Am Med Inform Assoc 2016;23(5):1007-15. DOI PubMed

41. Dorr D, Bejan CA, Pizzimenti C, Singh S, Storer M, Quinones A. Identifying patients with significant problems related to social determinants of health with natural language processing. Stud Health Technol Inform 2019;264:1456-7. DOI PubMed

42. Carrell DS, Cronkite D, Palmer RE, Saunders K, Gross DE, Masters ET, Hylan TR, Von Korff M. Using natural language processing to identify problem usage of prescription opioids. Int J Med Inform 2015;84(12):1057-64.

DOI PubMed

43. Cacheda F, Fernandez D, Novoa FJ, Carneiro V. Early detection of depression: social network analysis and random forest techniques. J Med Internet Res 2019;21(6):e12554. DOI PubMed

44. Conway M, Hu M, Chapman WW. Recent advances in using natural language processing to address public health research questions using social media and consumer generated data. Yearb Med Inform 2019;28(1):208-17. DOI PubMed

45. Coppersmith G, Dredze M, Harman C. Quantifying mental health signals in Twitter. Proceedings of the Workshop on Computational Linguistics and Clinical Psychology: from linguistic signal to clinical reality. Baltimore (MA): 27 June 2014;p. 51-60. DOI

46. Gates A, Guitard S, Pillay J, Elliott SA, Dyson MP, Newton AS, Hartling L. Performance and usability of machine learning for screening in systematic reviews: a comparative evaluation of three tools. Syst Rev 2019;8(1):278. DOI PubMed

47. Przybyła P, Soto AJ, Ananiadou S. Identifying personalised treatments and clinical trials for precision medicine using semantic search with Thalia. Manchester (UK): TREC; 2017.

48. Bannach-Brown A, Przybyła P, Thomas J, Rice AS, Ananiadou S, Liao J, Macleod MR. Machine learning algorithms for systematic review: reducing workload in a preclinical review of animal studies and reducing human screening error. Syst Rev 2019;8(1):23. DOI PubMed

49. Norman C, Leeflang M, Spijker R, Kanoulas E, Névéol A. A distantly supervised dataset for automated data extraction from diagnostic studies. ACL Workshop on Biomedical Natural Language Processing, Florence (IT): 2019 Aug. DOI

50. Tsafnat G, Glasziou P, Karystianis G, Coiera E. Automated screening of research studies for systematic reviews using study characteristics. Syst Rev 2018;7(1):64. DOI PubMed
51. Lerner I, Créquit $P$, Ravaud $P$, Atal I. Automatic screening using word embeddings achieved high sensitivity and workload reduction for updating living network meta-analyses. J Clin Epidemiol 2019;108:86-94. DOl PubMed

52. Tucker TC, Durbin EB, McDowell JK, Huang B. Unlocking the potential of population-based cancer registries. Cancer 2019;125(21):3729-37. DOI PubMed

53. Mohammadhassanzadeh $\mathrm{H}$, Sketris I, Traynor R, Alexander S, Winquist B, Stewart SA. Using natural language processing to examine the uptake, content, and readability of media coverage of a pan-Canadian drug safety research project: cross-sectional observational study. JMIR Form Res 2020;4(1):e13296. DOl PubMed

54. Banerji A, Lai KH, Li Y, Saff RR, Camargo CA Jr, Blumenthal KG, Zhou L. Natural language processing combined with ICD-9-CM codes as a novel method to study the epidemiology of allergic drug reactions. J Allergy Clin Immunol Pract 2020;8(3):1032-1038.e1. DOI PubMed

55. Young IJ, Luz S, Lone N. A systematic review of natural language processing for classification tasks in the field of incident reporting and adverse event analysis. Int J Med Inform 2019;132:103971. DOI PubMed

56. Henry S, Buchan K, Filannino M, Stubbs A, Uzuner O. 2018 $\mathrm{n} 2 \mathrm{c} 2$ shared task on adverse drug events and medication extraction in electronic health records. J Am Med Inform Assoc 2020;27(1):3-12. DOI PubMed

57. Fan B, Fan W, Smith C, Garner H. Adverse drug event detection and extraction from open data: a deep learning approach. Inf Process Manage 2020;57(1):102131. DOI

58. Yu W, Zheng C, Xie F, Chen W, Mercado C, Sy LS, Qian L, Glenn S, Tseng HF, Lee G, Duffy J, McNeil MM, Daley MF, Crane B, McLean HQ, Jackson LA, Jacobsen SJ. The use of natural language processing to identify vaccine-related anaphylaxis at five health care systems in the Vaccine Safety Datalink. Pharmacoepidemiol Drug Saf 2020;29(2):182-8. DOI PubMed

59. Liu F, Weng $\mathrm{C}, \mathrm{Yu} \mathrm{H}$. Advancing clinical research through natural language processing on electronic health records: traditional machine learning meets deep learning. In: Richesson RL, Andrews JE, editors: Clinical Research Informatics. Springer International Publishing; 2019. p. 35778. DOI

60. Chan L, Beers K, Yau AA, Chauhan K, Duffy Á, Chaudhary K, Debnath N, Saha A, Pattharanitima P, Cho J, Kotanko P, Federman A, Coca SG, Van Vleck T, Nadkarni GN. Natural language processing of electronic health records is superior to billing codes to identify symptom burden in hemodialysis patients. Kidney Int 2020;97(2):383-92. DOI PubMed

61. Juhn Y, Liu H. Artificial intelligence approaches using natural language processing to advance EHR-based clinical research. J Allergy Clin Immunol 2020;145(2):463-9. DOI PubMed 
62. Wang $Y$, Wang L, Rastegar-Mojarad M, Moon S, Shen F, Afzal N, Liu S, Zeng Y, Mehrabi S, Sohn S, Liu H. Clinical information extraction applications: A literature review. J Biomed Inform 2018;77:34-49. DOI PubMed

63. Laranjo L, Dunn AG, Tong HL, Kocaballi AB, Chen J, Bashir R, Surian D, Gallego B, Magrabi F, Lau AY, Coiera E. Conversational agents in healthcare: a systematic review. $J$ Am Med Inform Assoc 2018;25(9):1248-58. DOI PubMed

64. Head to health. COVID-19 support. Department of Health; Australian Government (accédé 2020-01-27). https:// headtohealth.gov.au/sam-the-chatbot

65. Pereira J, Díaz Ó. Using health chatbots for behavior change: a mapping study. J Med Syst 2019;43(5):135. DOI PubMed

66. Dion M, AbdelMalik P, Mawudeku A. Les données massives et le Réseau mondial d'information en santé publique (RMISP). Rele-vé des maladies transmissibles au Canada 2015;41(9):241-7. DOI

67. Ghosh S, Chakraborty P, Lewis BL, Majumder M, Cohn E, Brownstein JS, Marathe M, Ramakrishnan N. GELL: Automatic extraction of epidemiological line lists from open sources. Proceedings of the 23rd ACM SIGKDD International Conference on Knowledge Discovery and Data Mining; 2017 Aug 13-17; Halifax (NS): Association for Computing Machinery; 2017. p. 1477-86. DOI

68. Charles-Smith LE, Reynolds TL, Cameron MA, Conway M, Lau EH, Olsen JM, Pavlin JA, Shigematsu M, Streichert LC, Suda KJ, Corley CD. Using social media for actionable disease surveillance and outbreak management: a systematic literature review. PLoS One 2015;10(10):e0139701. DOI PubMed

69. Jordan S, Hovet S, Fung I, Liang H, Fu KW, Tsz Ho Tse Z. Using Twitter for public health surveillance from monitoring and prediction to public response. Data (Basel) 2018;4(1):6. DOI

70. Abbood A, Ullrich A, Busche R, Ghozzi S. EventEpi-A natural language processing framework for event-based surveillance medRxiv 2019;19006395. DOI

71. Anglin K. Gather-narrow-extract: A framework for studying local policy variation using web-scraping and natural language processing. J Res Educ Eff 2019;12(4):685-706. DOI

72. Tatman R, Conner K. Effects of talker dialect, gender \& race on accuracy of Bing speech and YouTube automatic captions. Proc Interspeech 2017;934-8: DOI

73. Spasic I, Nenadic G. Clinical text data in machine learning: systematic review. JMIR Med Inform 2020;8(3):e17984. DOI PubMed

74. Rajkomar A, Hardt M, Howell MD, Corrado G, Chin MH. Ensuring fairness in machine learning to advance health equity. Ann Intern Med 2018;169(12):866-72. DOI PubMed
75. Gramlich J. 10 facts about Americans and Facebook. Washington (DC): Pew Research Center (accédé 2020-0127). https://www.pewresearch.org/fact-tank/2019/05/16/ facts-about-americans-and-facebook/

76. Xu C, Doshi T. Fairness indicators: scalable infrastructure for fair ML system. Mountain View (CA): Google (accédé 202001-27). DOI

77. Holstein K, Vaughan JW, Daumé H, Dudík M, Wallach $H$. Improving fairness in machine learning systems: What do industry practitioners need? $\mathrm{CHI}$ <19: Proceedings of the $2019 \mathrm{CHI}$ Conference on Human Factors in Computing Systems. 2019 Paper No.: 600. p. 1-16. DOI

78. Wiens J, Price WN 2nd, Sjoding MW. Diagnosing bias in data-driven algorithms for healthcare. Nat Med 2020;26(1):25-6. DOI PubMed

79. Chen IY, Joshi S, Ghassemi M. Treating health disparities with artificial intelligence. Nat Med 2020;26(1):16-7. DOI PubMed

80. Montreal Declaration for a Responsible Development of Artificial Intelligence. Forum on the Socially Responsible Development of Al: 2017 Nov 2-3: Montréal (QC) (accédé 2020-01-18). https://www.montrealdeclaration-responsibleai. com/the-declaration

81. Secrétariat du Conseil du Trésor du Canada. Directive sur la prise de décision automatisée. Ottawa (ON) : Gouvernement du Canada (mise à jour 2019-02-05; accédé 2020-01-27). https://www.tbs-sct.gc.ca/pol/doc-fra.aspx?id=32592

82. Friedman C, Rindflesch TC, Corn M. Natural language processing: state of the art and prospects for significant progress, a workshop sponsored by the National Library of Medicine. J Biomed Inform 2013;46(5):765-73. DOI PubMed

83. Sheikhalishahi S, Miotto R, Dudley JT, Lavelli A, Rinaldi F, Osmani V. Natural language processing of clinical notes on chronic diseases: systematic review. JMIR Med Inform 2019;7(2):e12239. DOI PubMed

84. Ford E, Curlewis K, Wongkoblap A, Curcin V. Public opinions on using social media content to identify users with depression and target mental health care advertising: mixed methods survey. JMIR Ment Health 2019;6(11):e12942. DOl PubMed

85. Radebaugh C, Erlingsson U. Introducing tensorflow privacy: learning with differential privacy for training data. Medium. com (accédé 2020-01-27). https://medium.com/tensorflow/ introducing-tensorflowprivacy-learning-with-differential -privacy-for-trainingdata-b143c5e801b6

86. Penning de Vries BB, van Smeden M, Rosendaal FR, Groenwold RH. Title, abstract, and keyword searching resulted in poor recovery of articles in systematic reviews of epidemiologic practice. J Clin Epidemiol 2020;121:55-61. DOl PubMed

87. Obermeyer Z, Powers B, Vogeli C, Mullainathan S. Dissecting racial bias in an algorithm used to manage the health of populations. Science 2019;366(6464):447-53. DOI PubMed 
88. Coronavirus tech handbook: natural language processing. https://coronavirustechhandbook.com/nlp

89. COVID-19 Open Research Dataset Challenge (CORD-19): An Al challenge with Al2, CZI, MSR, Georgetown, NIH \& The White House. San Francisco (CA): kaggle.com (accédé 202001-27). https://www.kaggle.com/allen-institute-for-ai/CORD19-research-challenge

90. PubMed Central. Public Health Emergency COVID-19 Initiative. Bethesda (MD): US National Library of Medicine (accédé 2020-01-27). PubMed

91. Bullock J, Luccioni A, Pham KH, Lam CS, Luengo-Oroz $M$. Mapping the landscape of artificial intelligence applications against COVID-19. arXiv:2003.11336 [cs.CY]. https://vectorinstitute.ai/wp-content/uploads/2020/03/ arxiv-mappingai.pdf

92. Allen Institute for Artificial Intelligence (AI2). CORD-19 Explorer: explore the dataset. https://cord-19.apps.allenai. org/
93. Chen E, Lerman K, Ferrara E. COVID-19: the first public coronavirus Twitter dataset. Ithaca (NY): Cornell University (accédé 2020-01-27). https://arix.org/abs/2003.07372

94. LitCovid. Bethesda (MD): U.S. National Library of Medicine (accédé 2020-01-27). https://www.ncbi.nlm.nih.gov/research/ coronavirus/

95. Coronafiles: Chatbots take strain off Denmark's emergency helplines (accédé 2020-01-27). https://sifted.eu/articles/ coronafiles-chatbots-helplines/

96. Kritikos M. At a glance: scientific foresight: What if we could fight coronavirus with artificial intelligence? Scientific Foresight Unit, European Parliament. https://www.europarl. europa.eu/RegData/etudes/ATAG/2020/641538/EPRS_ ATA(2020)641538_EN.pdf

97. Against Al. COVID-19 Canada. About us. Toronto (ON): CIFAR (accédé 2020-01-27). https://ai-against-covid.caa

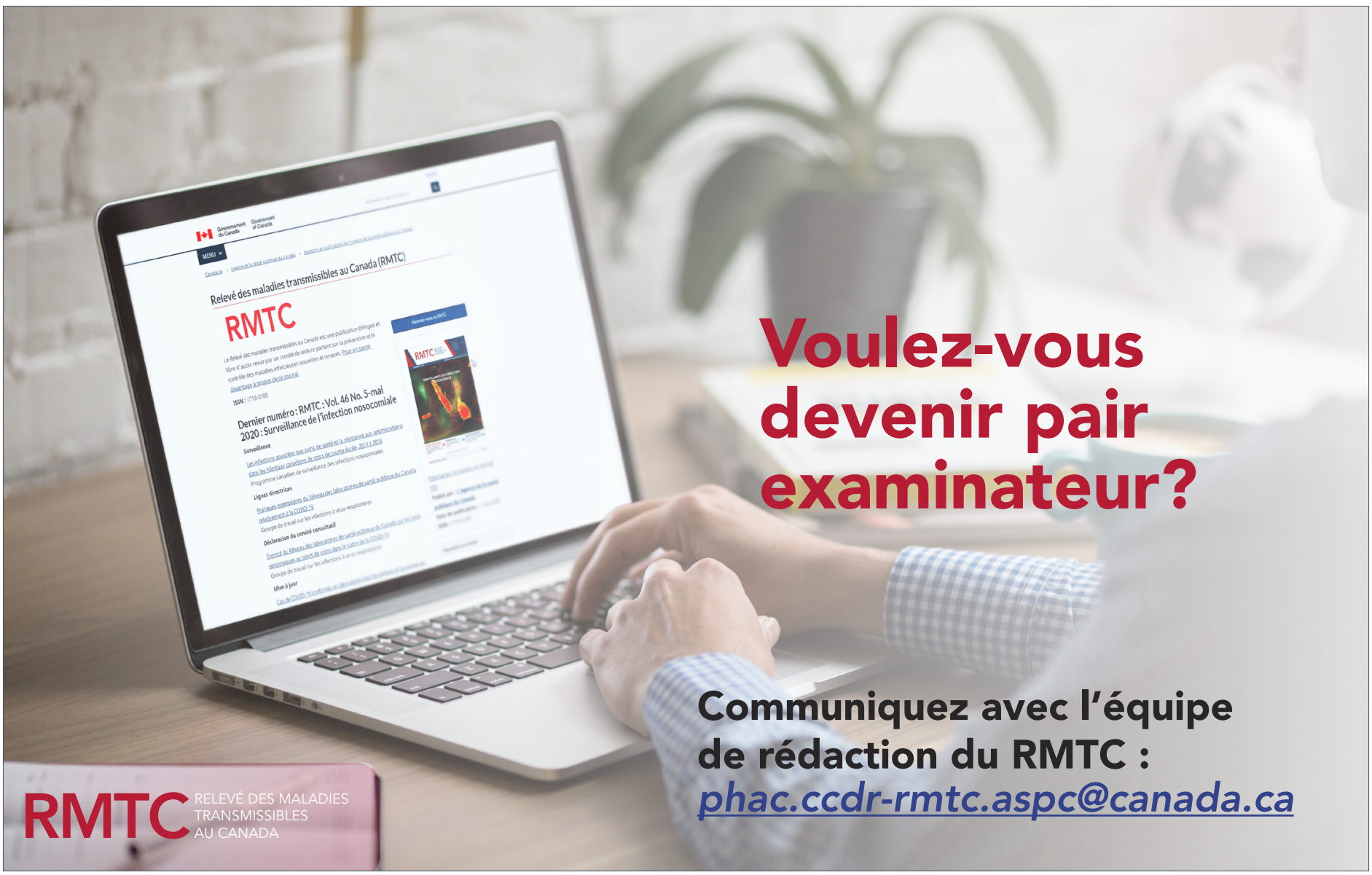

